\title{
Clouds over the summertime Sahara: an evaluation of Met Office re- trievals from Meteosat Second Generation using airborne remote sensing
}

John C. Kealy et al.

Correspondence to: John C. Kealy (j.kealy@exeter.ac.uk)

The copyright of individual parts of the supplement might differ from the CC-BY 3.0 licence. 
Kealy et al.: (Supplimentary) Performance of Meteosat cloud retrievals over the Sahara: a comparison with airborne data
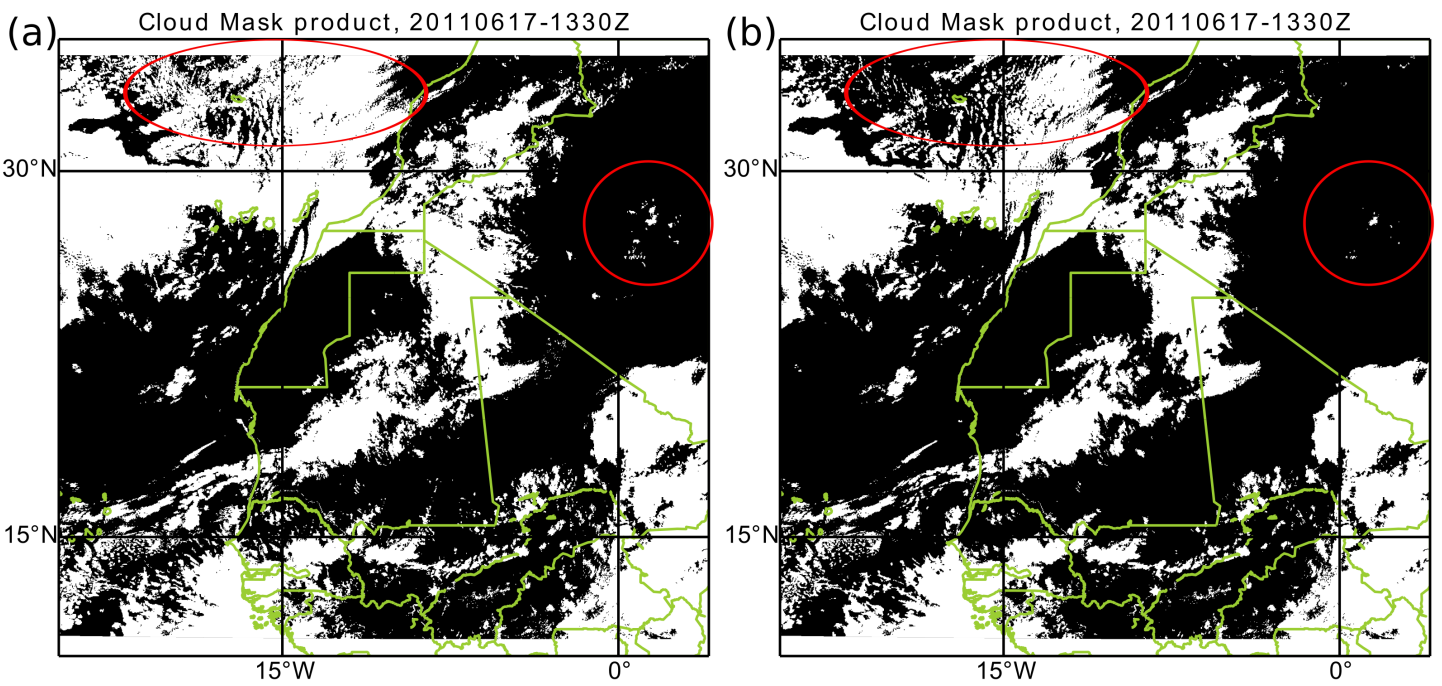

Figure S1. Sample Met Office cloud mask product showing a) AllCloud mask and b) CloudRetrieval mask during flight B600. Pixels with fractional cloud amounts will be represented as cloudy more readily by the AllCloud, leading to a greater number of cloud contaminated pixels in each frame relative to the CloudRetrieval mask. The CloudRetrieval mask presents a more conservative selection of cloudy pixels. Areas highlighted in red show the most prominent differences between these images. 


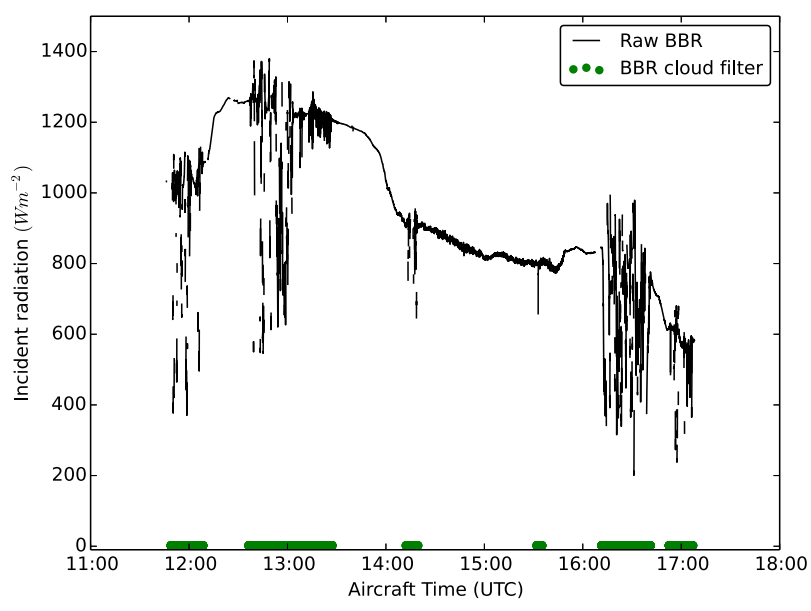

Figure S2. Sample of the cloud filter (shown in green just above the $\mathrm{x}$-axis) for removing cloud above the aircraft, derived from the pyranometer (BBR), shown for the time-series of flight B699 (6 June 2012). The filter is based on the large rate of change in BBR signal which occurs when solar radiation passes through cloud.

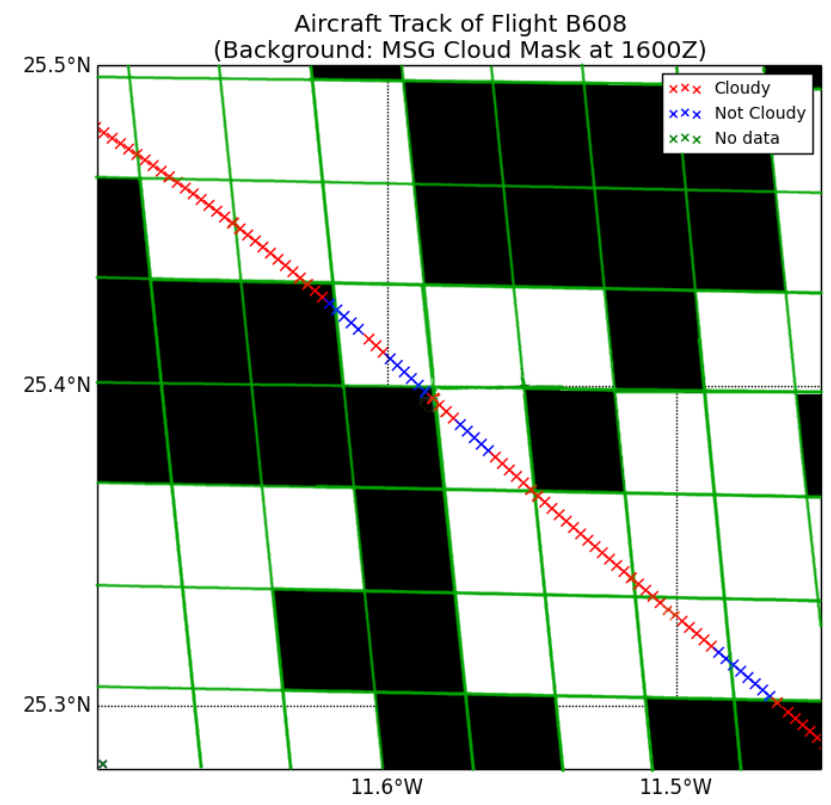

Figure S3. Zoomed-in segment of the B608 flight track (22 June 2011), showing the relative resolution of the SEVIRI pixels (black/white) against the aircraft resolution (red/blue).

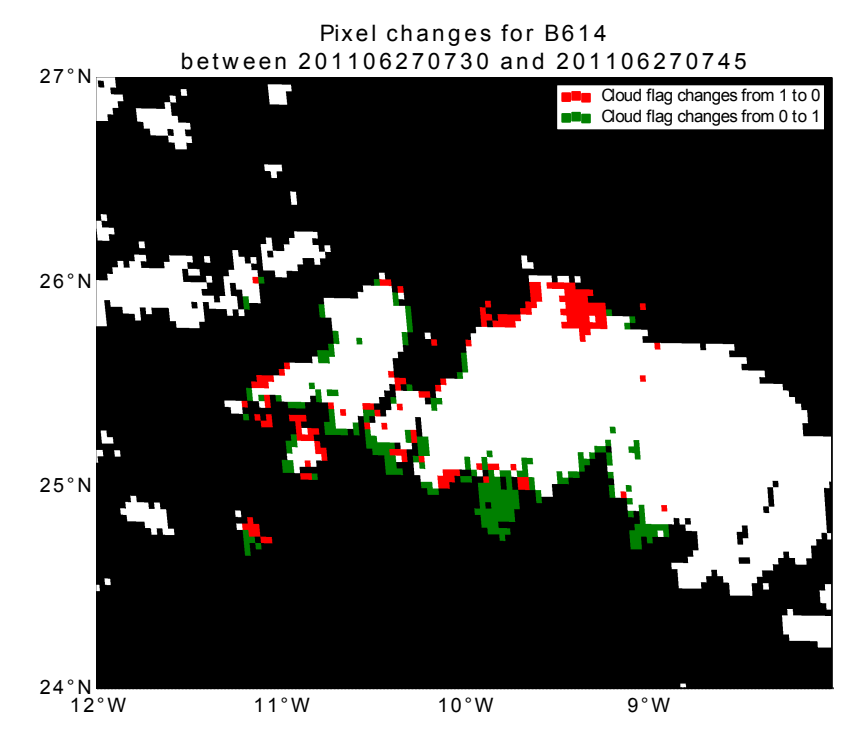

Figure S4. Sample image of the scale of change in the cloud mask over the 15 minute SEVIRI acquisition time, illustrating the method for calculating the temporal matching uncertainty (Sect. 2.5). The edges of cloudy areas are the most prone to changing their cloud mask value. 
Kealy et al.: (Supplimentary) Performance of Meteosat cloud retrievals over the Sahara: a comparison with airborne data
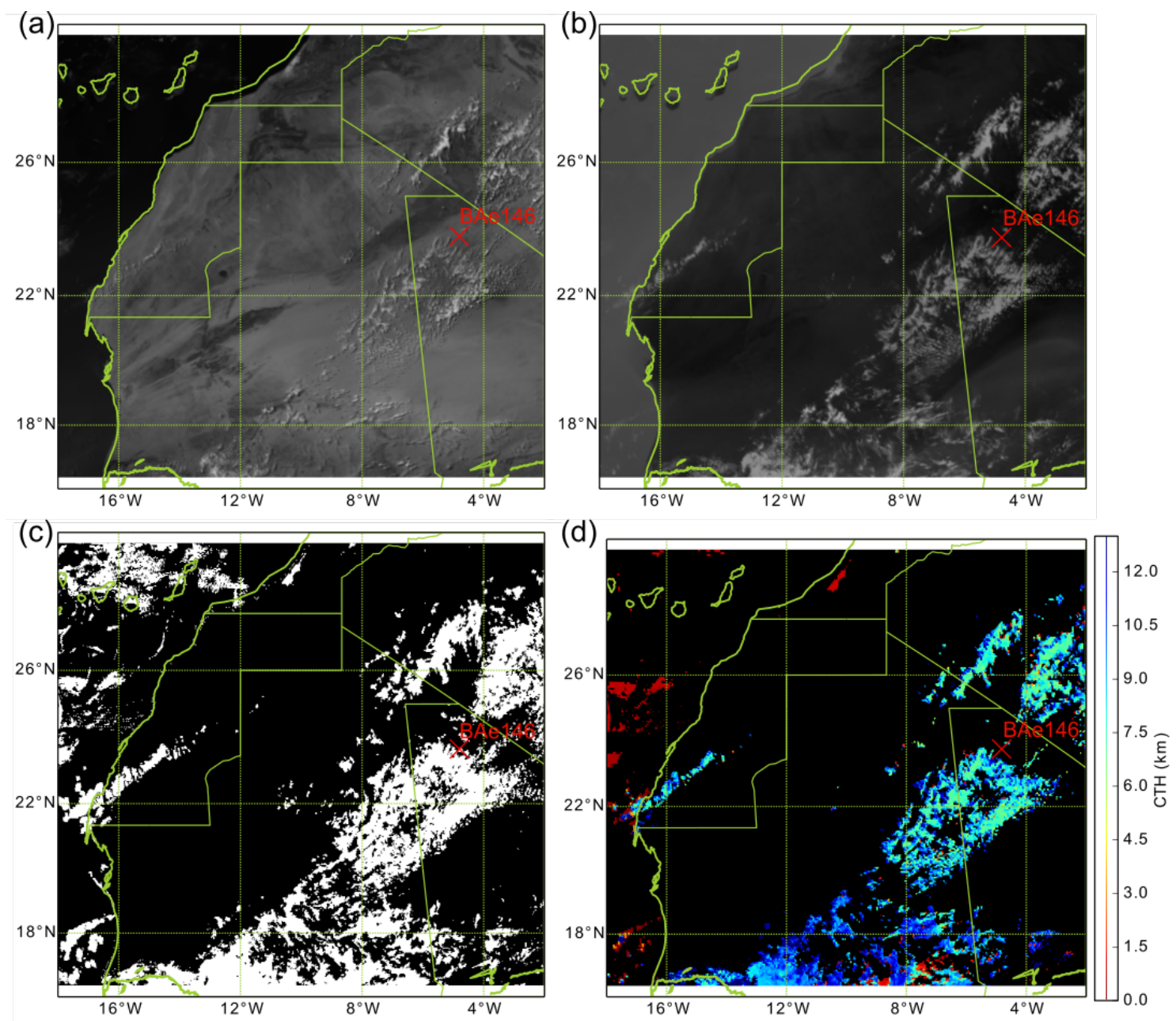

Figure S5. SEVIRI imagery to compliment the cloud scene shown in Fig. 4a (Flight B706, 14 Jun 2012 at 1500 UTC) for (a) Visible channel (b) IR channel (c) AllCloud cloud mask and (d) CTH. The position of the BAe146 is shown in red. 
Kealy et al.: (Supplimentary) Performance of Meteosat cloud retrievals over the Sahara: a comparison with airborne data
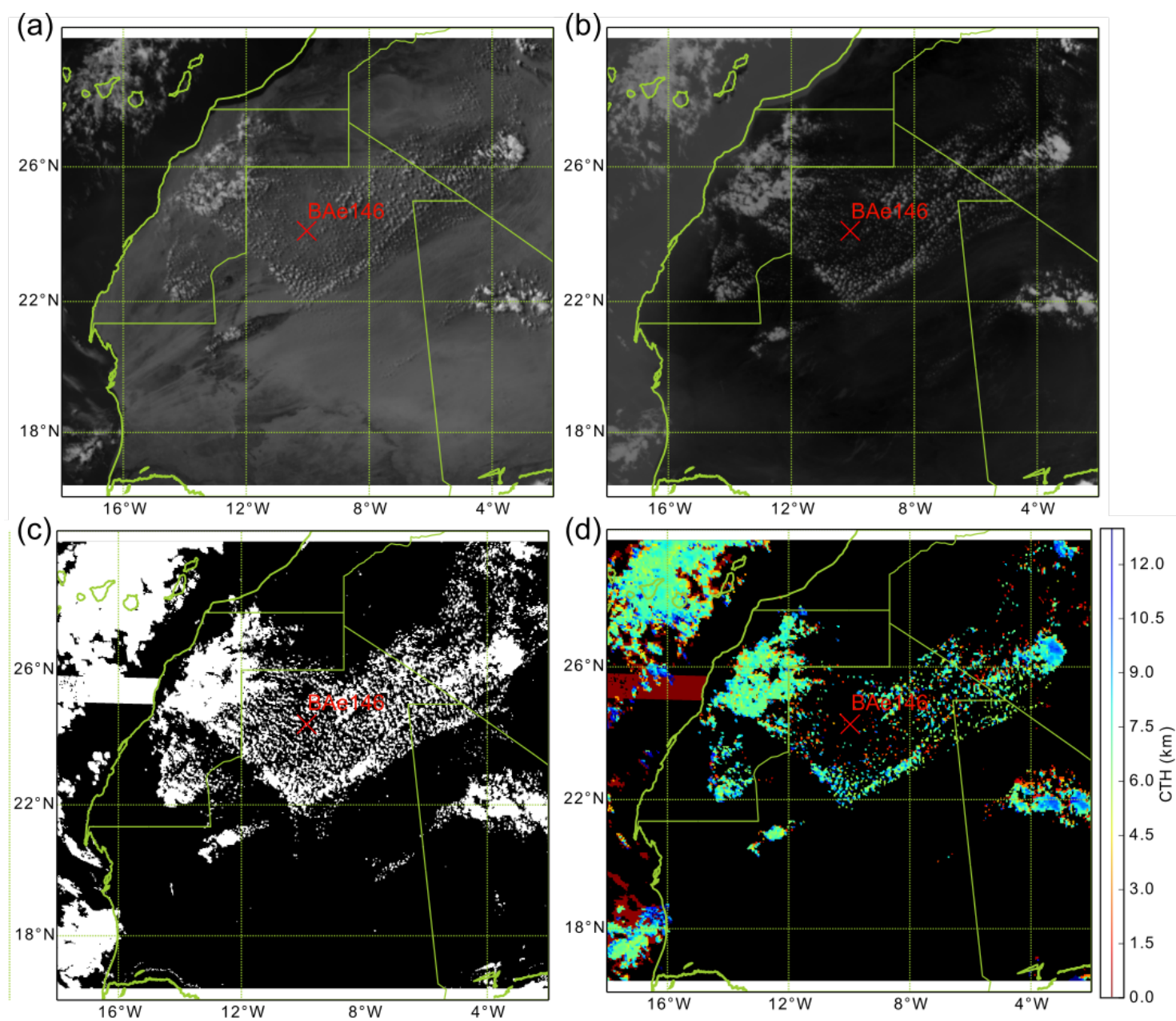

Figure S6. As Fig. S5 but corresponding to Fig. 4b (Flight B608, 22 Jun 2011 at 1615 UTC) 